\title{
Antenna Efficiency Calculations for Electrically Small, RFID Antennas
}

\author{
Amir Galehdar, David V. Thiel, and Steven G. O'Keefe
}

\begin{abstract}
Radio frequency identification (RFID) antenna efficiency is an important component of link budget design. A method of moments technique based on the summation of segment currents compares favorably with two different results obtained using the finite element method (radiation pattern integration and Wheeler cap). The efficiency of a resonant dipole was found to be proportional to the inverse square root of the conductivity. For a typical RFID meander line antenna in free space the relationship is more severe.
\end{abstract}

Index Terms-Antenna efficiency, numerical electromagnetic code (NEC), radio frequency identification (RFID), Wheeler cap, wire antennas.

\section{INTRODUCTION}

$\mathbf{T}$ HE concept of radio frequency identification (RFID) was introduced when the first paper on modulated backscatter (basic principle of passive RFID tag) was published [1]. There has been rapid development in recent years as the technique is now used in many commercial applications such as access control, animal tracking, security, and toll collection. A typical RFID transponder (tag) which can be passive (no battery) or active (with battery) consists of an antenna and an integrated circuit chip which is capable of storing an identification number and other information.

In a passive backscatter RFID tag, the querying transmitted signal from the reader consists of modulated information with periods of unmodulated carrier which is converted to electrical power to run the circuit [2]. The power stored can be estimated from the Friis free-space formula assuming other factors such as the electromagnetic characteristics of materials near or in contact with tag are ignored [3]. The "read-range" of the RFID tag is one vital parameter used to assess tag performance, and is defined as the maximum distance at which RFID reader can detect the backscattered signal from the tag [4]. In order to maximize the read range, high-gain tags are required, however, due to space limitations, the antennas usually need to be relatively small. As a general guide, most RFID systems are electrically small; i.e., $k_{r}=0.1$ where $k$ is wavenumber and $r$ is the radius of a sphere enclosing the antenna. These antennas are often heavily loaded with passive components in order to reduce the resonant frequency, however, this technique can reduce the antenna efficiency and gain. An antenna with low efficiency caused by poor conductivity of material can severely limit the reading range of the RFID system [5].

Manuscript received November 17, 2006; revised January 9, 2007.

The authors are with the Centre for Wireless Monitoring and Applications, Griffith University, Nathan, Queensland 4111, Australia.

Color versions of one or more of the figures in this letter are available at http://ieeexplore.ieee.org.

Digital Object Identifier 10.1109/LAWP.2007.891960
In this letter, three methods of calculating the efficiency for RFID antennas using computational electromagnetic methods are reviewed. The efficiency of electrically small wire antennas located in free space was calculated using:

- pattern integration using the finite element method (HFSS);

- Wheeler cap method using the finite element method (HFSS); and

- loss method using a method of moments calculation [numerical electromagnetic code (NEC)].

The Wheeler cap method is the most common experimental method of finding the efficiency in practice [6], [7]. HFSS simulation software was used to model the Wheeler cap technique in addition to efficiencies calculated by pattern integration, the default method in HFSS [8]. The loss method developed in this study is based on a calculation of the loss in each segment of the antenna with the aid of the current distribution calculated using NEC software [9]. These three methods were used to calculate the efficiency of a dipole and a meander RFID antenna with different track conductivity values at a resonant frequency of $869 \mathrm{MHz}$, the RFID standard frequency for Europe.

\section{THEORY}

\section{A. Pattern Integration}

In this method, the radiation efficiency is measured as a ratio of radiated power to antenna input power. In HFSS, the effect of the local environment can be included in the calculation, however, only the free space results are presented in this paper. The radiated power $P_{\text {rad }}$ is measured by dividing a bounding sphere $s$ in the far field of the antenna into small segments $d s$ and integrating the time-average power from each segment over the far field surface area. This can be expressed by

$$
P_{\text {rad }}=\Re \int_{s} E \times H^{*} . d s
$$

where $\Re$ is the real part of the a complex number, $E$ is the radiated electric field at the surface, and $H^{*}$ is the complex conjugate of $H$ the radiated magnetic field at the surface.

The accepted input power $P_{\text {in }}$ is the power entering the structure through all the ports. For a one-port radiating structure in HFSS, any impedance mismatch in the feed structure is ignored. This is calculated using

$$
P_{\text {in }}=\Re \int_{A} E \times H^{*} . d s
$$


where $A$ is the input feed area [8]. The radiation efficiency $\eta$ is calculated from

$$
\eta=\frac{P_{\mathrm{rad}}}{P_{\mathrm{in}}} .
$$

While this is a very common method of calculating the efficiency, it is not always reliable because it is based on the computed radiated power. It is possible that the computed radiated power is slightly different to the actual radiation power $[8, \mathrm{ch}$. 15-p. 46].

\section{B. Wheeler Cap}

This method is based on distinguishing between the loss resistance $R_{l}$ and the input resistance $R_{\text {in }}$ by assuming that the antenna can be modeled as a series of parallel $R L C$ circuit at or close to the resonant frequency. Equation (3) can be modified and $\eta$ calculated using

$$
\eta=\frac{P_{\text {rad }}}{P_{\text {in }}}=\frac{P_{\text {rad }}}{P_{\text {rad }}+P_{\text {loss }}}
$$

where $P_{\text {loss }}$ is the power loss in the structure due to resistance, ground loss, etc. The input power can be written

$$
P_{\text {in }}=\frac{1}{2} R_{\text {in }} I^{2}
$$

where $R_{\text {in }}$ is the input resistance (radiation plus loss resistance), and $I$ is the current of the modeled $R L C$ circuit. The power loss $P_{\text {loss }}$ is

$$
P_{\text {loss }}=\frac{1}{2} R_{l} I^{2}
$$

Equation (4) can be written

$$
\eta=\frac{\frac{1}{2} R_{\mathrm{in}} I^{2}-\frac{1}{2} R_{l} I^{2}}{\frac{1}{2} R_{\mathrm{in}} I^{2}}=\frac{R_{\mathrm{in}}-R_{l}}{R_{\mathrm{in}}} .
$$

$P_{\text {in }}$ can be determined but $P_{\text {loss }}$ and $P_{\text {rad }}$ are more difficult. Wheeler [7] suggested that one can eliminate the radiation resistance from the real part of input impedance by enclosing the antenna with a conducting sphere of radius of $1 / k$. Based on this theory, the input impedance was determined once when the structure is located in free space $\left(Z_{\text {in }}\right)$, and once when it is covered by the conductive cap $\left(Z_{w c}\right)$. The efficiency calculation becomes

$$
\eta=\frac{\Re\left(Z_{\text {in }}\right)-\Re\left(Z_{w c}\right)}{\Re\left(Z_{\text {in }}\right)} .
$$

There were modifications made to this method after suggestions that not all antennas can be modeled as $R L C$ circuits, however a wire antenna in free space at resonant frequency is suitable for this method of approach [10]. This method is usually used for practical measurements using a real antenna and a network analyzer. In this letter, the method was used in the HFSS simulation software.

\section{NEC Efficiency}

The NEC is based on the method of moments [9]. It is fast and reliable, although most versions do not routinely provide radiation efficiency in the output file. One method of calculating the antenna efficiency from NEC results is to use the pattern integration method as explained in the previous section, however this is not always accurate. Another approach is to calculate the power lost in the structure and compare it to input power. This method is highly dependent on the structure and the surroundings, but it can be implemented quite simply for wire antennas in free space.

If the current is assumed to be uniform and the only loss in the structure is the resistive loss $R_{l}$ then [11]

$$
R_{l}=R_{s} \frac{\ell}{2 \pi a}
$$

where

$$
R_{s}=\sqrt{\frac{\pi f \mu_{0}}{\sigma}}
$$

and $R_{s}$ is surface resistance, $a$ is the radius of the wire, $f$ is the frequency, $\mu_{0}$ is the permittivity of free space, $\sigma$ is the conductivity of the conductor, and $\ell$ is the length of the wire. The efficiency becomes

$$
\eta=\frac{R_{\text {in }}-R_{l}}{R_{\text {in }}}=\frac{R_{r}}{R_{r}+R_{l}}=\frac{1}{1+\frac{R_{l}}{R_{r}}}
$$

where $R_{r}$ is the radiation resistance. Combining these equations gives

$$
\begin{aligned}
\eta & =\frac{1}{1+\frac{\frac{\ell}{2 \pi a} \sqrt{\frac{\pi f \mu_{0}}{\sigma}}}{R_{r}}}=\left(1+\frac{\ell}{2 \pi a R_{r}}\left(\frac{\pi f \mu_{0}}{\sigma}\right)^{\frac{1}{2}}\right)^{-1} \\
& =\left(1+\left(\frac{\ell^{2} f \mu_{0}}{4 \pi a^{2} R_{r}^{2}}\right)^{\frac{1}{2}} \sigma^{-\frac{1}{2}}\right)^{-1}
\end{aligned}
$$

If $\sigma$ is sufficiently large, one can apply the binomial approximation to obtain:

$$
\eta=1-\left(\frac{\ell^{2} f \mu_{0}}{4 \pi a^{2} R_{r}^{2}}\right)^{\frac{1}{2}} \sigma^{-\frac{1}{2}}+\ldots \Rightarrow \eta \propto \sigma^{-\frac{1}{2}}
$$

Even with very small wire antennas, the current is not uniform over the entire structure so a numerical method of calculating the current distribution is required. In this case NEC was used calculate the current distribution on each segment of wire in the antenna. If there are $N$ segments in the NEC model of the complete antenna and the wire segment length is small enough, the current over the length of the $i$ th segment $i_{i}$, is approximately constant. The total power loss $P_{\text {loss }}$ is the sum of the losses in each segment:

$$
P_{\text {loss }}=\sum_{i=1}^{N} \frac{1}{2} R_{i} i_{i}^{2}
$$

where $R_{i}$ is segment resistance.

Assuming the $i$ th segment has length $\ell_{i}$, and using (7) we can write

$$
\eta=\frac{R_{\mathrm{in}} I^{2}-\sqrt{\frac{\pi f \mu_{0}}{\sigma}} \sum_{i=1}^{N} \frac{\ell_{i} i_{i}^{2}}{2 \pi a_{i}}}{R_{\mathrm{in}} I^{2}}
$$




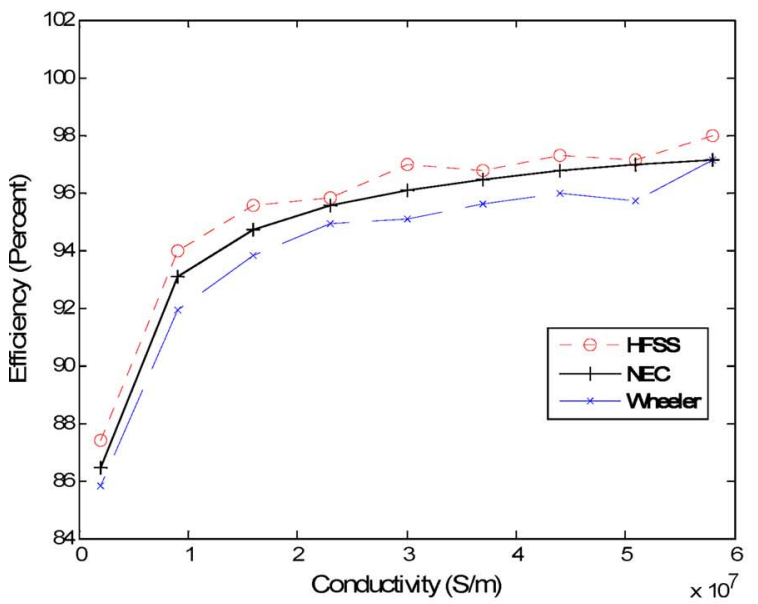

(a)

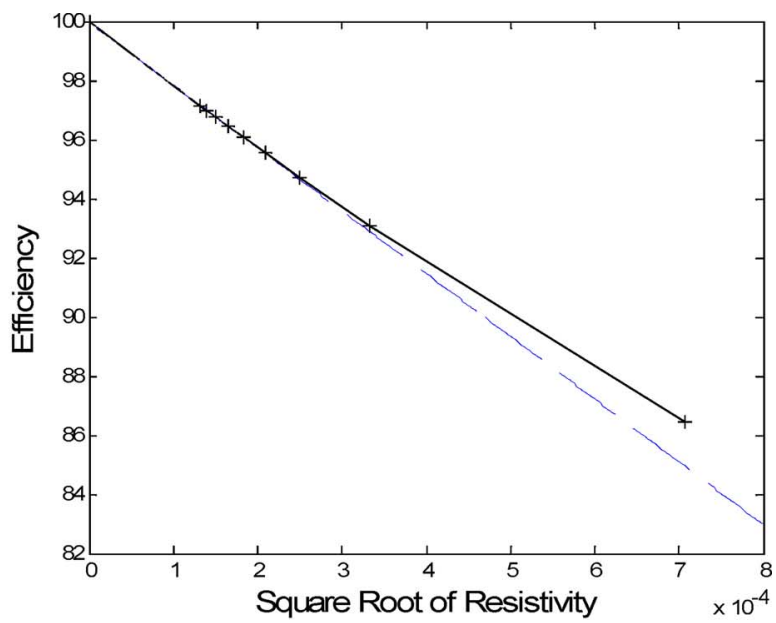

(b)

Fig. 1. (a) The efficiency verses dipole conductivity at $869 \mathrm{MHz}$. (b) Efficiency versus the square root of resisitivity.

where $a_{i}$ is the diameter of the $i$ th segment. If all segment lengths in the model are equal $\left(\ell_{i}=\ell\right)$ and the radius $\left(a_{i}=a\right)$ is unchanged throughout the structure, (15) can be written as

$$
\eta=\frac{R_{\mathrm{in}} I^{2}-\sqrt{\frac{\pi f \mu_{0}}{\sigma}} \frac{\ell}{2 \pi a} \sum_{i=1}^{N} i_{i}^{2}}{R_{\mathrm{in}} I^{2}} .
$$

\section{RESULTS}

\section{A. Dipole}

A 166-mm long, center-fed, dipole antenna with a wire radius of $0.05 \mathrm{~mm}$ resonates at $869 \mathrm{MHz}$. Assuming the wire has a circular cross section, the NEC results were calculated using 167 equally sized segments. As the HFSS meshing uses triangular elements, a wire with a square cross section was used. In order that the surface area was identical in both models, a rectangular to round wire ratio of 0.59 was used. Fig. 1 shows good agreement between all three methods. In Fig. 1(b), the resistivity $(=1 / \sigma)$ was plotted rather than the conductivity so that a perfect conductor value could be included on the graph.

Conductivity values from $2 \times 10^{6} \mathrm{~S} / \mathrm{m}$ (the value of RFID silver ink [3] $)$ to copper $\left(5.8 \times 10^{7} \mathrm{~S} / \mathrm{m}\right)$ were calculated. The relationship plotted in Fig. 1(b) is approximately linear for $\sigma>$ $10^{7} \mathrm{~S} / \mathrm{m}$. This agrees with (13). While the current distribution on the dipole is not uniform, changes in the current due to changes in conductivity scale linearly.

The computation time using NEC was the smallest of the three methods investigated. The pattern integration method using HFSS required more than 500 times the time for the NEC calculation. The Wheeler cap method needs two HFSS runs and so required an even longer time.

\section{B. Meander Line Dipole}

The meander line dipole antenna was designed for RFID application in [4] which was $40 \mathrm{~mm}$ long and $28 \mathrm{~mm}$ wide with $a=0.05 \mathrm{~mm}$. The exact dimensions and configuration of the antenna are shown in Fig. 2.

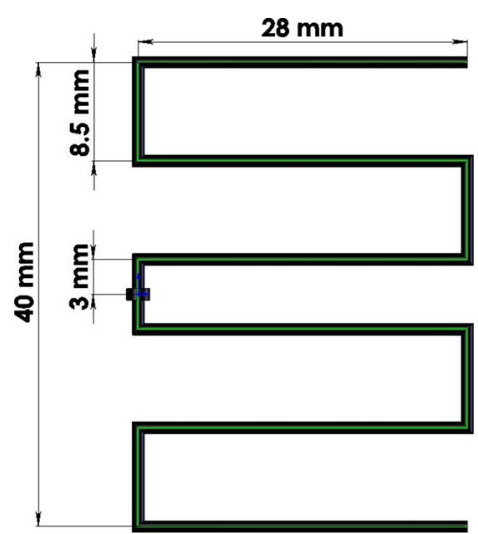

Fig. 2. Dimensions of the meander line dipole antenna (after [3]).

The efficiency was calculated using three different methods and ten different values of conductivity. Fig. 3 shows consistency from the loss method (NEC) and both the pattern integration method (HFSS) and the Wheeler cap method (HFSS). The Wheeler cap method has more variation. To maximize the accuracy all 15 meshing passes on HFSS were applied to the structure [8].

The NEC and Wheeler cap results show 100\% efficiency for a perfectly conducting line. The HFSS pattern integration result was $102 \%$, a slight over estimate probably due to an inaccurate absorbing boundary condition. In Fig. 3(b), the efficiency decreases far more rapidly with resistivity compared to the dipole results in Fig. 1(b) so that (13) is no longer applicable.

\section{CONCLUSION}

For a straight line dipole antenna, the antenna efficiency was linearly proportional to the square root of the resistivity, however the decrease in efficiency for the meander line is far more dramatic. Calculations using other meander line dipole structures showed similar results. It is critical in fabricating RFID antennas that the conductivity of the dipole is as high as possible. 


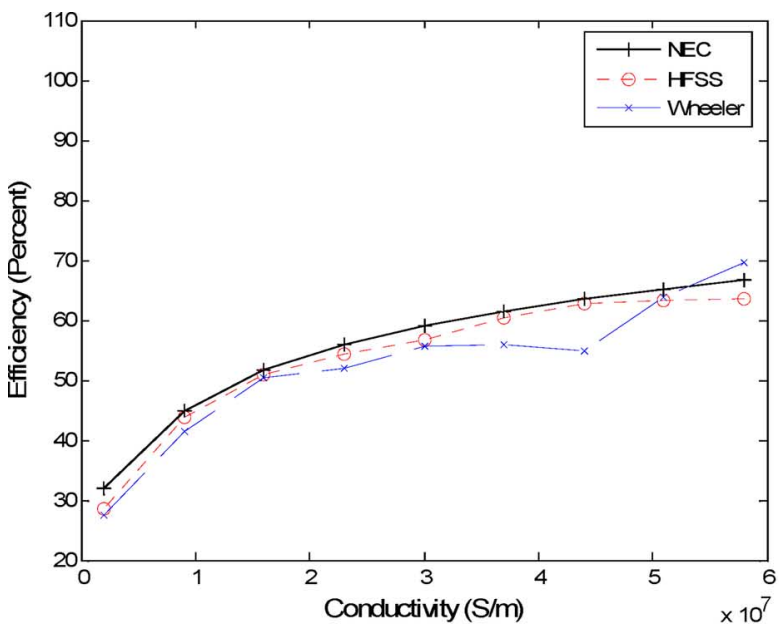

(a)

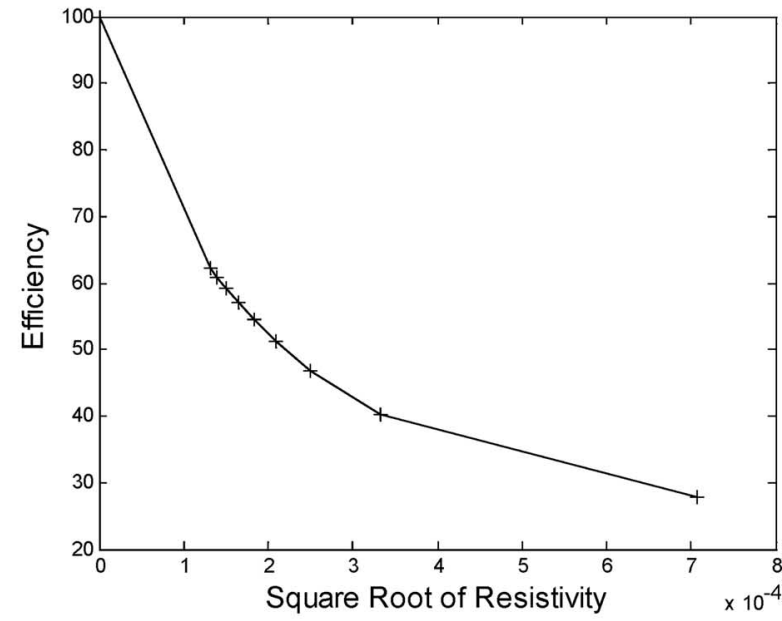

(b)

Fig. 3. Meander line dipole efficiency. (a) The efficiency verses the conductivity. (b) Efficiency versus the square root of resistivity.

The loss method based on NEC calculated currents is computationally efficient and more accurate in some cases compared to the HFSS methods. The technique results in a smoother plot of efficiency versus conductivity indicating improved accuracy.

\section{REFERENCES}

[1] K. Finkenzeller, RFID Handbook: Fundamentals and Applications in Contactless Smart Cards and Identification, 2nd ed. New York: Wiley, 2003.

[2] K. V. Seshagiri Rao, P. V. Nikitin, and S. F. Lam, "Antenna design for UHF RFID tags: A review and a practical application," IEEE Trans. Antennas Propag., vol. 53, pp. 3870-3876, 2005.

[3] J. D. Griffin, G. D. Durgin, A. Haldi, and B. Kippelen, "RF tag antenna performance of various materials using radio link budgets," IEEE Trans. Antenna Wireless Propag. Lett., vol. 5, pp. 247-250, 2006.
[4] G. Marrocco, "Gain-optimized self-resonant meander line antenna for RFID application," IEEE Trans. Antenna Wireless Propag. Lett., vol. 2, pp. 302-305, 2003.

[5] P. V. Nikitin, S. Lam, and K. V. Rao, "Low cost silver ink RFID tag antennas," in IEEE Antennas Propag. Soc. Int. Symp., 2005, vol. 2B, pp. 353-356.

[6] E. H. Newman, P. Bohley, and C. H. Walter, "Two methods for the measurement of antenna efficiency," IEEE Trans. Antennas Propag., vol. 23, pp. 457-461, 1975.

[7] H. A. Wheeler, "The radiansphere around a small antenna," Proc. IRE, pp. 1325-1331, Aug. 1959.

[8] HFSS 9.1 Hfss Onlinehelp Ansoft Corp., (undated).

[9] “NEC-Win Plus, User's Manual,” Nittany Scientific Inc., 2003.

[10] W. E. McKinzie, III, "A modified Wheeler cap method for measuring antenna efficiency," in IEEE Antennas Propag. Soc. Symp., Jul. 1997, pp. 542-545.

[11] D. K. Cheng, Field and Wave Electromagnetics, 2nd ed. Reading, MA: Addison-Wesley, 1989. 